\title{
An Accurate Physical Model for PV Modules with Improved Approximations of Series-Shunt Resistances
}

\author{
José Maurilio Raya-Armenta ${ }^{1}$, Pablo R. Ortega ${ }^{2}$, Najmeh Bazmohammadi ${ }^{1}$, Sergiu V. Spataru ${ }^{3}$, \\ Juan C. Vasquez ${ }^{1}$, and Josep M. Guerrero ${ }^{1}$ \\ ${ }^{1}$ Department of Energy Technolgy, Center for Research on Microgrids (CROM), Aalborg University, Denmark, Aalborg, 9220 (e-mail: \\ $\{$ jmra, naj, juq, joz\}@et.aau.dk). \\ ${ }^{2}$ Universitat Politècnica de Catalunya, Micro and NanoTechnologies (MNT) group, Jordi Girona 1-3 Modul C-4, E - 08034 Barcelona, \\ Spain (e-mail: pablo.rafael.ortega@upc.edu). \\ ${ }^{3}$ Department of Photonics Engineering, Technical University of Denmark, Denmark, 4000 Roskilde (e-mail: sersp@fotonik.dtu.dk).
}

\begin{abstract}
An accurate model to represent the photovoltaic modules is essential to facilitate the efficient deployment of these systems in terms of design, analysis, and monitoring considerations. In this respect, this study proposes a new approach to improve the accuracy of the widely-used five-parameter single-diode model. Two new physical equations are introduced to represent the series and shunt resistances while the other parameters are represented by well established physical expressions. In the proposed model, most of the parameters are in terms of the cell temperature, irradiance, and datasheet values, while a few parameters need to be tuned. The model is compared with four well-known methodologies to extract the parameters of the single-diode and double-diode models. The simulation studies make use of the different I-V characteristics provided in the PVs' datasheets, characteristics extracted from an outdoor module, as well as the ones simulated with the software PC1D. The results show an improved precision of the proposed model to estimate the power characteristics for a wide range of temperatures and irradiances, not only in the MPP, but also in the whole range of voltages. Furthermore, the proposed physical model can be easily applied to other kind of studies where a physical meaning of the PV parameters is of great importance.
\end{abstract}

Keywords: Photovoltaic, translating equations, shunt resistance, series resistance, bandgap energy, single-diode model, physical modelling.

\section{Introduction}

The photovoltaic (PV) systems are one of the most promising technologies to produce green electricity and contribute to slow down the climate change due to their reliability and unlimited availability of sun [5]. Thus, appropriate models to represent the PV modules are required to design, monitoring, control, and operation management during their life times [36]. In this regard, the single-diode (SD) and double-diode (DD) models, which are two of the most widely-used models $[9,11,20,26,27]$, aim to estimate the actual PV-cell/module behavior not only under standard test conditions $(\mathrm{STCs})^{1}$, but also under any environmental condition.

Therefore, many strategies based on SD and DD models have been presented. Using analytical methods is a widelyused approach that usually makes assumptions that decrease the model accuracy, e.g. the ideal SD model [26,27]. Another technique is to deploy curve-fitting methodologies to match the measured maximum power point (MPP) with the value computed under STCs $[18,41]$. Although accurate results to model the current-voltage (I-V) characteristics are reported, inaccuracies still exist in points different from the MPP. An improved double parameter curve fitting method is presented in [37] to extract the initial parameters, while physical/empirical approximations are used to adjust the parameters to any condition. High accuracy in the whole I-V curve for different conditions of irradiance and temperatures above room temperature (RT) is observed while the parameters are bounded to values with a physical meaning. However, the shunt resistance is assumed to be constant while other studies suggest to consider its dependency on the irradiance and temperature $[14,24,32]$. Other studies solve a system of non-linear equations using the PV-module's datasheet values while using physical/empirical approximations to adjust the parameters to any condition $[12,35,39]$. These techniques are accurate in the MPP, but the solution is highly dependent on the initial guesses and it is quite likely to converge to a local minimum or even not converge. Likewise, a new technique is presented in [29] that uses datasheet information and an adaptive algorithm. Such a technique formulates the problem in the form of a constrained convex optimization problem with two decision variables. The results show a good enough accuracy above the RT while convergence to a unique solution is ensured. Nevertheless, the shunt resistance is expressed by an empirical approximation and the series resistance is considered constant when its dependency on the irradiance and temperature is emphasized in $[4,14,24]$. Other strategies are based on heuristic algorithms

\footnotetext{
${ }^{1}$ Irradiation $1000\left[\mathrm{~W} / \mathrm{m}^{2}\right]$, solar spectrum $A M 1.5 \mathrm{G}$, temperature $25\left[{ }^{\circ} \mathrm{C}\right]$.
} 


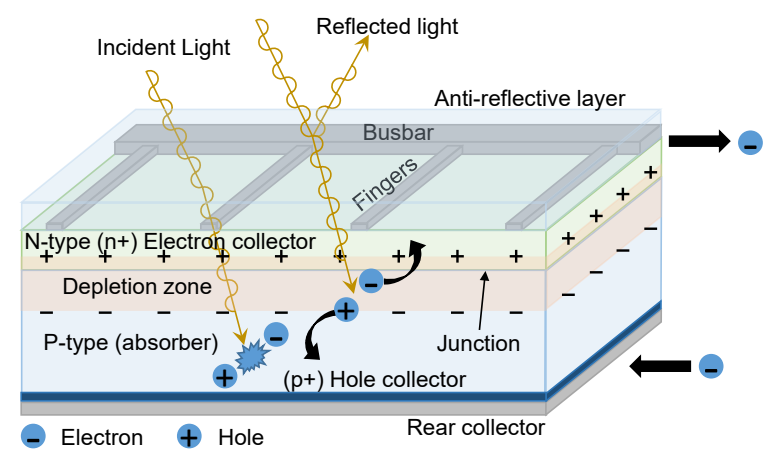

Figure 1: Structure of a standard Si PV-cell. Adapted from [16].

$[25,43,44]$, which are very accurate at any condition of irradiance and temperature, but they are slow and require a large measurement data set. Furthermore, artificial neural networks (ANNs) are proposed in $[6,20,28]$ to directly approximate the I-V characteristics or for computing the PV parameters. This technique could get similar accuracy to analytical methods without the need for mathematical formulations, but it requires a large set of measurement data for a specific module and the optimal ANN design could be different for each module.

Even though some of the existing techniques can provide good accuracy, expressions of several model parameters lack physical meaning, which limits their reliable application under different operating conditions. Therefore, such methods fail to accurately estimate the PV power in a wide range of temperature and irradiance or predict the PV-cell/module behavior. Moreover, the empirical models should be reshaped considerably, if not totally, for their application in other kind of studies. On the other hand, a physical model can be easily applied to other studies, e.g. degradation of PV-cells due to bombarding of energetic particles and photon recycling. Additionally, physics-based models could be used for modeling degradation process, diagnosing faults, and preventive and corrective maintenance. Further, the physical parameters might help PV-cell designers to optimize the PV-modules in terms of price, efficiency, and lifetime.

In this regard, several attempts have been made during the last years to introduce a physical meaning for the PV parameters. In [38], a good accuracy of power estimation is presented in four different PV-modules for the whole range of voltage variation $\left(0-V_{o c}\right.$ (open circuit voltage) ) at different irradiance conditions and temperatures above RT. However, the expressions for the series and shunt resistances are still semi-empirical, which might limit the physical representation of the PV-cell/module. A similar study is introduced in [30], but considering a DD configuration. The results show a good accuracy in two different PV-modules for the whole range of voltage at different irradiance conditions and temperatures above RT. However, this method has also the limitation of using semi-empirical approximations for the resistances. In [15], it is proposed to estimate the PV-cell parameters taking advantage of the dark I-V curve's derivative using a triple diode model. The results show good fitting accuracy with the experimental dark I-V curve. However, the study is limited to test just one kind of cell under darkness and under a specific temperature.

Therefore, the need for a PV model with four main features, namely high accuracy in a wide range of temperature and irradiance, low processing time, use of limited experimental data, and physical expressions of the PV-cell/module parameters still exists. In this respect, this paper proposes a new modelling technique that introduces new physical approximations for the series and shunt resistances, which depend on the irradiance, cell temperature, and a few tuning parameters; considers the narrowing effect in the bandgap energy due to the heavy doping; and uses physical expressions for the ideality factor, reverse saturation current, and photo-generated current. The goal is to increase the accuracy of the SD model in a wide range of irradiance and temperature conditions; give a physical description to the series-shunt resistances while using well-established physical expressions for the other parameters to reduce computational burden; and use the PV panel's datasheet information, which could ease the model deployment in practical applications where measurement data may not be available.

To verify the effectiveness of the proposed model, it is compared with four well-established techniques to extract the parameters of PV-modules. For this purpose, datasheet/experimental data of five different PV-modules is used. The results prove superiority of the proposed modelling approach in terms of accuracy over a wide range of temperature and irradiance throughout the whole range of voltage $\left(0-V_{o c}\right)$.

The rest of the paper is organized as follows. Section 2 presents the SD and DD models and some widely-used techniques for the parameters extraction. Section 3 introduces the new approximations for the series-shunt resistances as well as the bandgap energy. Section 4 gives a summary of the modelling procedure. Experimental results are discussed in section 5. And finally, concluding remarks are given in Section 6.

\section{The Photovoltaic-Cell Models}

A PV-cell based on mainstream silicon technology is fundamentally comprised of $n$-type and $p$-type semiconductor wafers, collectors, and anti-reflective coating, Fig. 1. The SD and DD models, which are widely-used to represent the PV system behavior are introduced in this section. Besides, four widely-used techniques to compute the parameters of such models are presented, which will be used to compare the performance of the proposed models in Section 5. 


\subsection{Single-Diode Model}

This electrical circuit-based model is comprised of a current source to represent the photo-generated carriers; A series resistance to express losses caused by the load current; A shunt resistance to model the effect of the leakage current; And a diode to represent the diffusion and recombination [11,18,25,31], Fig. 2. Thus, assuming that all the cells of the PV-module are similar, the module behavior is given as

$$
I=I_{p h}-I_{0} \Pi(a)-\frac{V+I R_{s}}{R_{s h}},
$$

where $\Pi(a)=\exp \left(\frac{V+I R_{s}}{a}\right)-1$. Besides, $I_{p h}$ and $I_{0}$ give the equivalent photo-generated and reverse saturation current of the whole module, respectively. The equivalent series and shunt resistances for the whole module are given as $R_{s}$ and $R_{s h}$, respectively. And the equivalent ideality factor as $a=m V_{T}=m n_{s} k T / q$, where $k=8.62 \times 10^{-5}[\mathrm{eV} / \mathrm{K}]$ is the Boltzmann constant, $T$ is the cell temperature $[K], q=1.6021 \times 10^{-19}[C]$ is the elementary charge, $m$ is the ideality factor, and $n_{s}$ is the number of cells connected in series $[2,27,39,41]$.

\subsubsection{W. D. Soto Solution}

The widely-used translating equations that are proposed by [39] are

$$
\begin{aligned}
a & =a_{s t c} \frac{T}{T_{s t c}}, \\
I_{0} & =I_{0}^{s t c}\left(\frac{T}{T_{s t c}}\right)^{3} \exp \left[\frac{1}{k}\left(\left.\frac{E_{g}}{T}\right|_{s t c}-\frac{E_{g}}{T}\right)\right], \\
E_{g} & =E_{g}^{s t c}(1-0.0002677 \Delta T), \\
I_{p h} & =\frac{G}{G_{s t c}} \frac{M}{M_{s t c}}\left(I_{p h}^{s t c}+\alpha_{i s c} \Delta T\right),
\end{aligned}
$$

where $\Delta T=T-T_{s t c}, E_{g}$ is the bandgap energy (1.12 [eV] for Si-based cells at STC), $G$ is the irradiance $\left[\frac{W}{m^{2}}\right], M$ is an air mass modifier, and $\alpha_{i s c}$ is the short-circuit temperature coefficient. Further, the series and shunt resistances are expressed as $R_{s}=R_{s}^{s t c}$ and $R_{s h}=R_{s h}^{s t c} \frac{G_{s t c}}{G}$, respectively. The reference parameters (at STCs) are found by solving a system of non-linear equations.

\subsubsection{G. Villalva Solution}

This strategy is introduced in [41]. The technique consists in increasing the series resistance while the shunt resistance is updated accordingly. The goal is to match the computed maximum power with the experimental value provided in the datasheet at STCs. The translating equations for this technique are

$$
\begin{aligned}
I_{p h} & =\left(I_{p h}^{s t c}+\alpha_{i s c} \Delta T\right) \frac{G}{G_{s t c}}, \\
I_{p h}^{s t c} & =\frac{R_{s h}+R_{s}}{R_{s h}} I_{s c}^{s t c}, \\
I_{0} & =\frac{I_{s c}^{s t c}+\alpha_{i s c} \Delta T}{\exp \left[\left(V_{o c}^{s t c}+\beta_{v o c} \Delta T\right) / a\right]-1},
\end{aligned}
$$

where $I_{s c}$ is the short-circuit current and $\beta_{v o c}$ is the temperature coefficient of the $V_{o c}$. In addition, $R_{s h}$ is expressed in terms of $R_{s}$ using (1) at STCs in the MPP, as follows

$$
R_{s h}=\frac{V_{m p}^{s t c}+I_{m p}^{s t c} R_{s}}{I_{p h}^{s t c}-I_{0}^{s t c} \exp \left[\left(V_{m p}^{s t c}+I_{m p}^{s t c} R_{s}\right) / a^{s t c}\right]+I_{0}^{s t c}-I_{m p}^{s t c}},
$$

where $V_{m p}$ and $I_{m p}$ correspond to the voltage and current at the MPP. After determining the resistances, they are kept constant. Recommended initial values for $R_{s h}$ and $R_{s}$ can be found in [41]. Furthermore, the ideality factor is arbitrarily chosen in the range $1.0 \leq m \leq 1.50$.

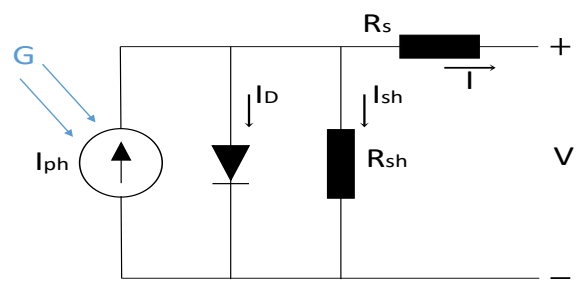

Figure 2: SD equivalent circuit model for a PV module. $V$ : Module voltage, $I$ : Module current. 


\subsubsection{Sera Solution}

Another solution is introduced in [35]. In this method, a system of three non-linear equations is solved by a numerical solver. The result provides the value of the series resistance, shunt resistance, and ideality factor, which are considered constant at any ambient condition. In addition, a system of equations describe $I_{0}, I_{p h}, I_{s c}$, and $V_{o c}$ at any condition of irradiance and temperature as

$$
\begin{aligned}
V_{o c}(T) & =V_{o c}^{s t c}+\beta v o c \Delta T \\
I_{s c}(T) & =I_{s c}^{s t c}+\alpha_{i s c} \Delta T \\
I_{0}(T) & =\left[I_{s c}(T)-\frac{V_{o c}(T)-I_{s c}(T) R_{s}}{R_{s h}}\right] \exp \left[-\frac{V_{o c}(T)}{a^{s t c}}\right], \\
I_{p h}(T) & =I_{0}(T) \exp \left[\frac{V_{o c}(T)}{a^{s t c}}\right]+\frac{V_{o c}(T)}{R_{s h}}, \\
I_{s c}(G) & =I_{s c}^{s t c}\left(G / G_{s t c}\right), \\
I_{p h}(G) & =I_{p h}^{s t c}\left(G / G_{s t c}\right), \\
V_{o c}(G) & =\ln \left|\frac{I_{p h}(G) R_{s h}-V_{o c}(G)}{I_{0}^{s t c} R_{s h}}\right| a^{s t c},
\end{aligned}
$$

where (16) should be determined by a numerical solver. The method suggests to follow the superposition principle to consider simultaneously the effect of irradiance and temperature in any parameter.

\subsection{Double-Diode Model}

This model is used to improve the accuracy of the SD model [13,34]. The second diode is located in parallel with the one in the SD model to represent the recombination in the depletion zone. The mathematical expression of this model for a module is given as

$$
I=I_{p h}-I_{01} \Pi\left(a_{1}\right)-I_{02} \Pi\left(a_{2}\right)-\frac{V+I R_{s}}{R_{s h}} .
$$

However, the improvement increases the complexity and the processing time [25].

\subsubsection{Z. Salam Solution}

This technique is introduced in [34] for a DD model. To reduce the computational burden to process the DD model parameters, an equal inverse saturation current in both diodes is assumed $I_{01}=I_{02}$. Besides, the ideality factor of $D_{1}$ and $D_{2}$ are set to $m_{1}=1$ and $m_{2} \geq 1.2$, respectively. Thus, only four parameters are left and the translating equations are

$$
\begin{aligned}
I_{p h} & =\frac{G}{G_{s t c}}\left(I_{p h}^{s t c}+\alpha_{i s c} \Delta T\right), \\
I_{01} & =I_{02}=\frac{I_{s c}^{s t c}+\alpha_{i s c} \Delta T}{\exp \left[\left(V_{o c}^{s t c}+\beta_{v o c} \Delta T\right) / V_{T}\right]-1} .
\end{aligned}
$$

$R_{s h}$ is expressed in terms of $R_{s}$ similarly to (9) but using (17) at STCs in the MPP. Recommended initial values for $R_{s h}$ and $R_{s}$ can be found in [41]. The goal is to match the computed MPP value with the value provided in the datasheet $P_{m p}^{s t c}$.

\section{A New Approximation of the Bandgap Energy, Series and Shunt Re- sistances}

\subsection{Bandgap Energy}

It has been shown that the bandgap energy decreases if the temperature increases. Besides, a narrowing effect is observed while the doping concentration of the impurities increases [19,23,40,42]. Thereby, the bandgap energy equation, along with a term $\Delta E_{g}$ to represent the narrowing effect, is given as

$$
E_{g}=E_{g, 0}-\frac{\alpha T^{2}}{T+\beta}-\Delta E_{g}
$$

where $E_{g, 0} \approx 1.169[\mathrm{eV}]$ is the bandgap energy at zero $K, \alpha \approx 4.9 \times 10^{-4}[\mathrm{eV} / K]$, and $\beta \approx 655[K]$ for Si $[40]$. Besides, for this study, $\Delta E_{g}$ is considered a tuning parameter taking into account that normally its value is in the order of $[m e V]$. 
Table 1: Assumptions of the proposed models

\begin{tabular}{ll}
\hline Parameter & Assumption \\
\hline Operation & The semiconductors comprising the PV-cell \\
& operates in the extrinsic stage. \\
Doping concentration & Non-degenerate semiconductor, \\
& $n \ll N_{c}$ and $p \ll N_{v}$. Besides, $N_{a}=N_{d}$. \\
Doping profile & Uniform along the PV-cell transverse section. \\
SRV & Not considered (not passivated). \\
$\tau_{r}$ & Constant. \\
Wafer thickness $(W)$ & $W=$ Constant $\forall$ layers and $W \gg$ Abs. Depth. \\
Injection level & Low. \\
Mobility ratio & $F_{\mu}=\mu_{p} / \mu_{n}=1$. \\
\hline SRV: Surface recombination velocity. $\tau_{r}:$ Mean recombination time.
\end{tabular}

\subsection{Series Resistance}

The series resistance is comprised of two parts, one belongs to the conductors and the other one to the semi-conductors.

\subsubsection{Resistance in Conductors}

Considering the range of temperatures on the earth surface, the series resistance of the conductive part is expressed as

$$
R_{s 1} \approx R_{s}^{s t c}\left(1+\alpha_{0} \Delta T\right)
$$

where $\alpha_{0}$ is the collectors temperature coefficient at $T_{\text {stc }}[33]$.

\subsubsection{Resistance in the Semi-Conductive Part}

The conductivity of a semiconductor is expressed as

$$
\sigma=q n \mu_{n}+q p \mu_{p}
$$

where $n$ and $p$ are the electron and hole densities, respectively. The corresponding mobilities are given as $\mu_{n}$ and $\mu_{p}[21]$

Carrier concentration In the extrinsic range, the carrier concentration will be comprised of the thermally generated and the photo-generated carriers. For the special case of an $n$-type semiconductor with a moderate-heavy doping $\left(N_{d}<10^{18}\left[1 / \mathrm{cm}^{3}\right]\right)$, the carriers concentration could be expressed as [21]

$$
\begin{aligned}
& n=N_{d}+\Delta n, \\
& p \approx \Delta n,
\end{aligned}
$$

where $N_{d}$ is the donors concentration and $\Delta n$ is the mean excess carrier concentration due to the photo-generation using the AM1.5G spectral irradiance and the wavelength from 280 to $1200[\mathrm{~nm}]$. Therefore, replacing $n$ and $p$ in (22) with (23) and (24), the conductivity will be expressed as

$$
\sigma_{n}=q \mu_{n} N_{d}\left[1+\frac{\Delta n}{N_{d}}\left(1+F_{\mu}\right)\right]
$$

where $F_{\mu}=\mu_{p} / \mu_{n}$. Likewise, the conductivity for the $p$-type semiconductor can be expressed as

$$
\sigma_{p}=q \mu_{p} N_{a}\left[1+\frac{\Delta n}{N_{a}}\left(1+F_{\mu}^{-1}\right)\right]
$$

where $N_{a}$ is the acceptors concentration and $\Delta n$ is defined as

$$
\Delta n=\frac{G \tau_{r}}{G_{s t c} h c W} \int_{A M 1.5 G} \eta_{\lambda} F_{\lambda} \lambda d \lambda
$$

where $\eta_{\lambda}=\frac{h c}{q \lambda} S R(\lambda)$ is the external quantum efficiency (EQE), $S R(\lambda)[A / W]$ is the spectral response, $h=6.626 \times$ $10^{-34}[\mathrm{Js}]$ is the Planck constant, and $c[\mathrm{~m} / \mathrm{s}]$ is the speed of light; $F_{\lambda}\left[\mathrm{Js}^{-1} \mathrm{~m}^{-3}\right]$ is the spectral irradiance; $W[\mathrm{~m}]$ is the wafer thickness; $\lambda[m]$ is the photon wavelength; $\tau_{r}[s]$ is the mean surface and bulk recombination time [3,7,21]. In this paper, two scenarios are analyzed: with $\Delta n$, and without $\Delta n$. Table 1 shows the assumptions made throughout the paper. 
Drift mobility The total mobility $\mu_{n}$ of electrons is obtained by using Matthiessen's rule as follows

$$
\frac{1}{\mu_{n}}=\frac{1}{\mu_{I}}+\frac{1}{\mu_{L}},
$$

where $\mu_{I}$ and $\mu_{L}$ show the mobility due to the ionized donor impurities and the lattice vibrations, respectively. Besides, the mobility is defined as $\mu \propto \tau=1 /\left(S v N_{s}\right)$, where $\tau$ is the mean free time between scattering events, $S$ is the cross section area of the scatterer, $v$ is the mean speed of the electrons in the conductive band (CB) (thermal velocity), and $N_{s}$ is the number of scatterers per unit volume [21].

Drift mobility due to lattice vibrations, $\mu_{L}$ : The scatterer cross-section area depends on the the atomic vibrations amplitude around the equilibrium point, which means $S \propto(3 / 2) k T$ [21]. Besides, it is considered that the electrons transferred to the $\mathrm{CB}$ will have a kinetic energy (KE) within $(3 / 2) k T$ and $(3 / 2) k T+\Delta E$. Thereby, the average total velocity of the electrons in the $\mathrm{CB}$ due to the action of temperature and energy gained from the photon is proposed to be

$$
v \propto\left(\frac{3}{2} k T+\alpha_{p h} \frac{G}{N}\right)^{1 / 2}
$$

where the first term represents the thermal $\mathrm{KE}$ and the second term represents the average KE gained from the absorbed photon. Besides, $\alpha_{p h}$ is the average ratio of the KE gained by an electron to the energy of the photon and $N$ is the global photon flux. $N_{s}$ is assumed to be constant. Accordingly, the drift mobility due to the lattice vibrations can be expressed as

$$
\begin{aligned}
\frac{1}{\mu_{L}} & \propto T g_{\alpha}^{1 / 2}, \\
g_{\alpha} & =\frac{3}{2} k T+\alpha_{p h} \frac{G}{N} .
\end{aligned}
$$

Drift mobility due to ionized impurities, $\mu_{I}$ : The scattering cross-section area in this mobility is related to the Coulombic attraction between the electrons in the CB and the ionized impurities. The scattering cross-section area is modified by considering that the KE in the electrons is comprised of the thermal excitation and the energy gained from the photon as

$$
S \propto g_{\alpha}^{-2}
$$

While the carrier velocity is kept as $v \propto g_{\alpha}^{1 / 2}$. Besides, the impurities concentration is considered to be constant. Thus, the drift mobility due to the ionized impurities is given as

$$
\frac{1}{\mu_{I}} \propto g_{\alpha}^{-3 / 2}
$$

Total mobility: Finally, using (28), the total mobility of the electrons can be calculated as follows

$$
\frac{1}{\mu_{n}}=\gamma_{n 1} g_{\alpha}^{-3 / 2}+\gamma_{n 2} T g_{\alpha}^{1 / 2},
$$

where $\gamma_{n 1}$ and $\gamma_{n 2}$ represent the proportional constants of the mobility due to the ionized impurities (33), and the mobility due to the lattice vibrations (30), respectively.

Resistance Considering the assumptions given in Table 1 , the resistance definition $R \propto 1 / \sigma$, the conductivity expression in (25), and the mobility (34), the resistance of an $n$-type semiconductor can be expressed as

$$
R_{n}=\left(\gamma_{n 1} g_{\alpha}^{-3 / 2}+\gamma_{n 2} T g_{\alpha}^{1 / 2}\right) /\left(1+2 \frac{\Delta n}{N_{d}}\right)
$$

Defining $\left.R_{n}\right|_{s t c}=R_{n}^{s t c}$, it is straightforward to drive the following equations from (35):

$$
\begin{aligned}
\frac{R_{n}}{R_{n}^{s t c}} & =\frac{\Gamma_{n 1} g_{\alpha}^{-3 / 2}+\Gamma_{n 2} T g_{\alpha}^{1 / 2}}{\Omega}, \\
\Gamma_{n_{2}} & =\frac{\Omega_{s t c}-\Gamma_{n 1} g_{\alpha, s t c}^{-3 / 2}}{T^{s t c} g_{\alpha, s t c}^{1 / 2}}, \\
\Omega & =1+2 \frac{\Delta n}{N_{d}}
\end{aligned}
$$


Table 2: Tuning parameters of the proposed model

\begin{tabular}{llc}
\hline Parameter & Description & Range \\
\hline $\mathrm{A}$ & Constant for $R_{s}$ & {$[0,1.5]$} \\
$\mathrm{B}$ & Constant for $R_{s h}$ & {$[0,1.5]$} \\
$\Gamma_{R}$ & Portion of $R_{s}$ (conductor part) & {$[0,1.0]$} \\
$\Delta E_{g}$ & Narrowing effect of $E_{g}$ & $\leq 90 \mathrm{meV}$ (poly) \\
& Ratio of the electron KE in the CB & $\geq 90 \mathrm{meV}$ (mono) \\
$\alpha_{p h}$ & to the photon energy & $(0,1.0)$ \\
\multicolumn{2}{l}{ The range for $A, B$, and $\Delta E_{g}$ are suggested based on the experience. }
\end{tabular}

where $\Gamma_{n 1}=\gamma_{n 1} / R_{n}^{s t c}$. Following the same procedure but using (26), the $p$-type resistance is expressed as

$$
\begin{aligned}
\frac{R_{p}}{R_{p}^{s t c}} & =\frac{\Gamma_{p 1} g_{\alpha}^{-3 / 2}+\Gamma_{p 2} T g_{\alpha}^{1 / 2}}{\Omega}, \\
\Gamma_{p_{2}} & =\frac{\Omega_{s t c}-\Gamma_{p 1} g_{\alpha, s t c}^{-3 / 2}}{T^{s t c} g_{\alpha, s t c}^{1 / 2}} .
\end{aligned}
$$

Considering $R_{s 2}=R_{p}+R_{n}, R_{p}=a R_{s 2}, R_{n}=b R_{s 2}$, and $a+b=1$, it is possible to derive an expression for $R_{s 2}$ as

$$
\frac{R_{s 2}}{R_{s}^{s t c}}=\left(\frac{\Omega_{s t c}}{\Omega}\right)\left[A \phi^{-3 / 2}+(1-A)\left(\frac{T}{T^{s t c}}\right) \phi^{1 / 2}\right],
$$

where $\phi=g_{\alpha} / g_{\alpha, s t c}$ and $A$ is a tuning parameter.

\subsubsection{Total Series Resistance}

The total series resistance can be expressed as follows using the conductive part represented in (21) and the semiconductive part given by (41).

$$
R_{s}=\Gamma_{R} R_{s 1}+\left(1-\Gamma_{R}\right) R_{s 2},
$$

where $\Gamma_{R}$ is a tuning parameter, which corresponds to the fraction of the total series resistance in the conductive part.

\subsection{Shunt Resistance}

For the PV modelling purpose, $R_{s h}$ is used to model the leakage current of the PV-modules, which flows across the crystal surface or through the grain boundaries for poly-crystalline (PC) technology instead of along the load [21]. $R_{s h}$ belongs to the semi-conductive part of the PV-module, thus the same approach proposed to drive (41) is adopted as

$$
\frac{R_{s h}}{R_{s h}^{s t c}}=\left(\frac{\Omega_{s t c}}{\Omega}\right)\left[B \phi^{-3 / 2}+(1-B)\left(\frac{T}{T^{s t c}}\right) \phi^{1 / 2}\right],
$$

where $B$ is the tuning parameter of the shunt resistance.

\section{The Proposed Single-Diode Model}

In addition to the expressions for $R_{s}, R_{s h}$, and $E_{g}$, the translating equations for $a, I_{0}$, and $I_{p h}$ are modeled using the proposed approach in [39] when air mass $M=M_{s t c}$. These expressions are given by (2), (3), and (5). If the temperature is measured at the back surface of the module, a transformation to the actual cell temperature is also needed $[1,45]$. In this paper, the following transformation is used

$$
T=T_{m}+\frac{G}{G_{s t c}} \Delta T
$$

where $T_{m}$ is the rear surface module temperature and $\Delta T$ is typically around $2 \sim 3\left[{ }^{\circ} \mathrm{C}\right]$ for flat plate modules [22]. However, in this study a better performance is observed for $\Delta T=4\left[{ }^{\circ} \mathrm{C}\right]$. Therefore, the translating equations of the proposed model are comprised of (2), (3), (5), (42), and (43) along with (20) and (44) for the bandgap energy and cell temperature, respectively. Table 2 shows the tuning parameters.

Similar to the other existing modelling approaches for PV-modules $[2,17,39]$, the proposed translating equations in this paper are a function of the corresponding parameter under the STC. To determine these parameters, this paper uses the teaching-learning based optimization (TLBO) algorithm. 


\section{Results and Discussion}

The proposed modelling methodology is validated for the PC and mono-crystalline (MC) PV technologies since they are widely-used nowadays. Two PC and one MC modules are studied using the I-V characteristics provided in their datasheets. A PC module located on the roof of the PV-Lab at Aalborg University is also studied using experimental $\mathrm{I}-\mathrm{V}$ curves. Another module is modeled with the software PC1D. Table 3 shows the information for each module. The parameters' values at STCs were computed by the TLBO algorithm and are used by the proposed model in this study and by the W. D. Soto solution [39].

The proposed model is considered without the excess of carriers due to the photo-generation effect (PGE) (Prop. 1) and with considering the excess of carriers (Prop. 2) as represented in (27). For comparison purposes, four widely-used techniques for PV modeling, which were introduced in Section 2, have been implemented in addition to the proposed method. The comparison is performed by using the mean absolute error in power (MAEP) introduced by $[37,38]$

$$
M A E P_{i}=\frac{\sum\left|P_{m e s, k}-P_{m o d, k}\right|}{N_{p}}, \quad k=1, \ldots, N_{p},
$$

where $N_{p}$ is the number of points on the power curve, $P_{m e s}$ is the PV power at a specific voltage from datasheet, and $P_{\text {mod }}$ is the power computed by the respective model. The evaluation of the MAEP is performed from 0 to $V_{o c}$ and around the MPP. Besides, the average MAEP is used as follows

$$
M A E P_{a v}=\frac{\sum M A E P_{i}}{N_{\text {curves }}}, \quad i=1, \ldots, N_{\text {curves }},
$$

where $N_{\text {curves }}$ is the total number of curves used. Further, the root mean square error (RMSE) of the power from $0 \mathrm{~V}$ to $V_{o c}$ and around the MPP is also analyzed. Table 4 shows the values for the tuning parameters used in this study. Furthermore, Prop. 2 requires additional data: The global spectral irradiance at AM1.5G given by the ASTM [10] to determine the total photon flux as $N \approx 2.90 \times 10^{21}\left[\# / \mathrm{sm}^{2}\right]$, the irradiance at the specific wavelength $G_{0, \lambda}\left[\mathrm{Js}^{-1} \mathrm{~m}^{2}\right]$ between 280 and $1200[\mathrm{~nm}]$; The spectral response of the MC and PC PV-cells to compute the EQE [8]; Cell thickness $W \approx 200[\mu \mathrm{m}] ;$ And the mean recombination time, $\tau_{r}=5 \times 10^{-5}[\mathrm{~s}]$. For all the modules, an average doping concentration of $N_{d}=10^{16}\left[\mathrm{~cm}^{-3}\right]$ is considered. Also, it is assumed that the fingers and busbars are made of Silver, $\alpha_{0} \approx 3.72 \times 10^{-3}\left[K^{-1}\right][33]$.

\subsection{MAEP and RMSE of power}

Firstly, the PV power characteristics of all modules are derived. Afterwards, the MAEP and RMSE of power are computed in the whole range of voltage from $0 \mathrm{~V}$ to $V_{o c}$ and in a few sampling points around the MPP. Fig. 3 represents the MAEP of all the models for the different modules at different conditions. The accuracy enhancement can be observed in Fig. 3-5, Fig. 3-6, and Fig. 3-10 when (27) is included. Table 5 presents the average MAEP of each model obtained for each of the modules at different levels of irradiance for the whole range of voltages and around the MPP. The results show the superiority of the proposed models Prop. 1 and Prop. 2 over the other models in the most of the conditions for all the modules in terms of the minimum average MAEP. Likewise, the average MAEP for the whole range of voltages and around the MPP at different levels of temperature is given in Table 6. It can be noticed that Prop. 1 outperforms other modelling approaches in reducing the average MAEP. In addition, a considerable difference between Prop. 1 and Prop. 2 is not observed.

Similarly, Table 7 shows the average RMSE of power obtained from each model for each of the modules in different irradiances. Likewise, Table 8 shows the average RMSE of power obtained from each model for each of the modules in different temperatures. The averages, in both tables, cover the whole range of voltages and around the MPP. The results once again show the superiority of the proposed models under different irradiance and temperature conditions.

\begin{tabular}{|c|c|c|c|c|c|}
\hline & KK280P & JAP60S01 & REC245PE & PC1D & M-60 \\
\hline \multicolumn{6}{|c|}{ Datasheet parameters } \\
\hline$I_{s c c}^{s t c}$ & 9.53 & 9.18 & 8.80 & 7.9755 & 9.08 \\
\hline $\begin{array}{l}s c \\
V_{o c}^{s t c}\end{array}$ & 38.9 & 38.17 & 37.10 & 39.324 & 37.90 \\
\hline$n_{s}$ & 60 & 60 & 60 & 60 & 60 \\
\hline$V_{m p}^{s t c}$ & 31.50 & 31.13 & 30.10 & 34.4085 & 30.80 \\
\hline$I_{m p}^{s t c}$ & 8.89 & 8.67 & 8.23 & 7.6232 & 8.60 \\
\hline$\alpha_{i s c}$ & 0.00559 & 0.00532 & 0.002112 & 0.000251 & 0.003632 \\
\hline$\beta_{\text {voc }}$ & -0.138 & -0.12596 & -0.10017 & -0.12416 & -0.12128 \\
\hline \multicolumn{6}{|c|}{ PV parameters under STCs (computed by the TLBO algorithm) } \\
\hline$I_{p h}^{s t c}$ & 9.52731 & 9.18789 & 8.69404 & 7.97837 & 9.07735 \\
\hline$I_{0}^{s t c}$ & 1.45198 & 0.17515 & 61.7734 & 0.06194 & 19.32055 \\
\hline$R_{s}^{s t c}$ & 0.27686 & 0.21724 & 0.26557 & 0.01382 & 0.15082 \\
\hline$R_{s h}^{s t c}$ & 190.645 & 501.121 & 2094.48 & 5000 & 594.029 \\
\hline$a^{\text {stc }}$ & 1.72293 & 1.54662 & 1.91299 & 1.53731 & 1.89903 \\
\hline
\end{tabular}

Table 3: PV-modules specifications 
Table 4: Tuning parameters used for each model at each PV-module

\begin{tabular}{lccccc}
\hline \multicolumn{5}{c}{ PV-models proposed (SD) } \\
\hline \multirow{5}{*}{ Param. } & KKalue used for each module \\
\cline { 2 - 6 } $\mathrm{A}$ & 1.004240 & 0.18 & 0.0064 & - & - \\
$\mathrm{B}$ & 0.516975 & 0.55 & 0.32 & 1.50 & 0.121 \\
$\Gamma_{R}$ & 0.954569 & 0.15 & 0.30 & 1.0 & 1.0 \\
$\Delta E_{g}$ & 80.645 & 32.25 & 70 & 6.5 & 223.75 \\
$\alpha_{p h}$ & 0.25 & 0.099 & 0.1485 & 0.03542 & 0.3264 \\
\hline \multicolumn{5}{c}{ Salam solution (DD) [34] } \\
\hline$m_{2}$ & 1.53 & 1.20 & 1.20 \\
\hline \multicolumn{5}{c}{ Villalva solution (SD) [41] } \\
\hline$m$ & 1.10 & 1.0 & 1.35 & 1.20 \\
\hline Note: $m_{2}$ is the ideality factor of diode $2 . \Delta E_{g}$ is in $[\mathrm{meV}]$.
\end{tabular}
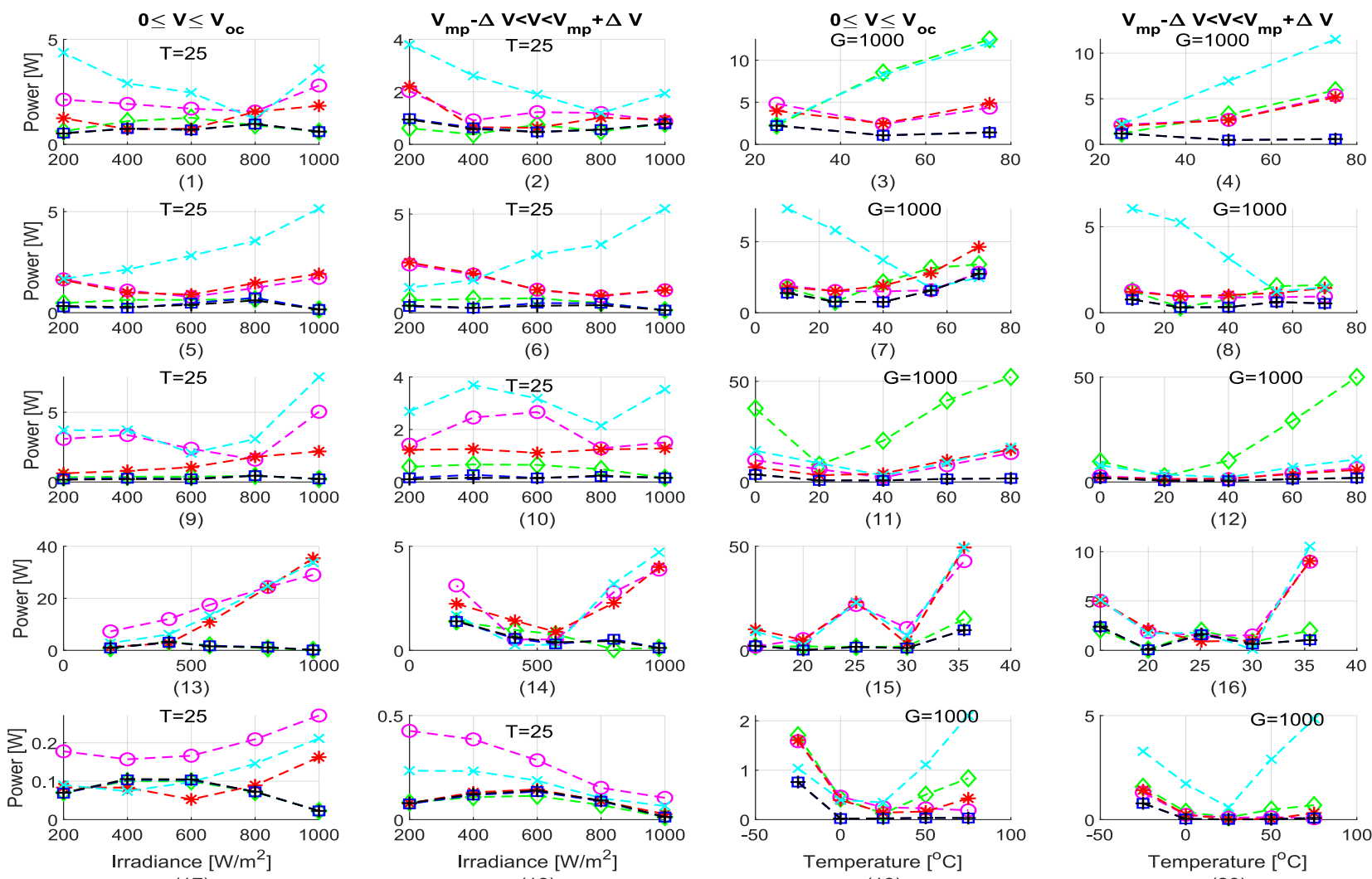

(17)
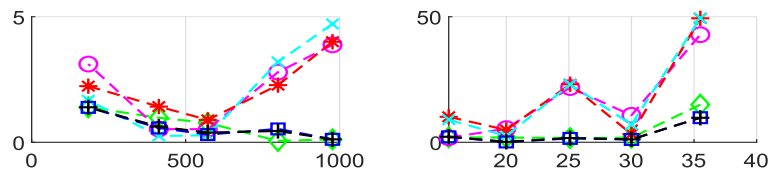

(15)

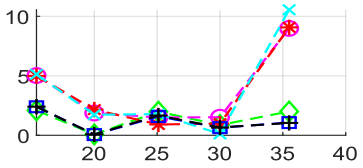

(16)

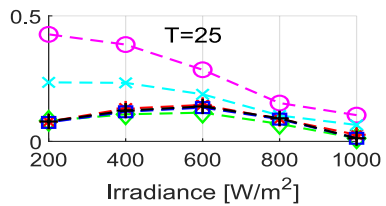

(18)

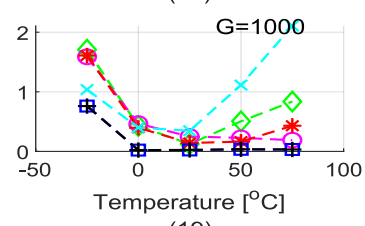

(19)

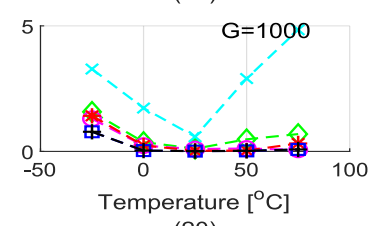

(20)

Figure 3: Diamond: D. Soto solution (SD); Circle: Salam solution (DD); Asterisk: Villalva solution (SD); Cross: Sera solution (SD); Square: Prop. 1 (SD); Plus: Prop. 2 (SD). From left to right. Column 1: MAEP for $0 \leq V \leq V_{o c}$ for different levels of irradiance. Column 2: MAEP around MPP for different levels of irradiance. Column 3: MAEP for $0 \leq V \leq V_{o c}$ for different levels of temperature. Column 4: MAEP around MPP for different levels of temperature. From top to bottom. Row 1: module KK280P3CD3CG. Row 2: module JAP60S01270SC. Row 3: module M60. Row 4: module REC245PE. Row 5: module PC1D. The points in (13)-(14), for each level of irradiance, correspond to rear surface temperatures of $\{25.9,25.3,24.1,25.1,25.3\}\left[{ }^{\circ} C\right]$, from left to right respectively. The points in (15)-(16) for each level of temperature correspond to irradiances of $\{205,255,842,219,1007\}\left[\mathrm{W} / \mathrm{m}^{2}\right]$, from left to right respectively.

\subsection{The Series and Shunt Resistances}

The mathematical representation of the resistances is derived by considering an $n-p$ junction. Therefore, the shunt resistance characteristics of a PV-module should be similar to the one of a semiconductor in the dark $(G=0)$, while the series resistance characteristics should be similar to the one of a linear combination between a semiconductor and a metal. Figure 4 shows $R_{s}$ of the PV-module JAP60S01-270-SC for both models: Prop. 1 and Prop. 2. According to Fig. $4, R_{s}$ at $G=0$ decreases when temperature increases. Besides, the effect of $\Gamma_{R}$ is clearly observed for large irradiances with a linear increment while the temperature increases. The shunt resistances have a similar behavior without the linear component.

When the photons hit the atoms inside the semiconductor lattice with enough energy $\left(G / N \geq E_{g}\right)$, the generation of electron-hole pairs starts and the carriers concentration density increases. The electrons "jump" from the valence band (VB) to the CB with an average velocity described by (29). Thereby, the increment of the carriers concentration and velocity will result in reducing the resistances of the semiconductors for a given temperature. The reduction is sharper for low temperatures, Fig. 4. However, the contribution of the metallic parts will increase the resistance while the temperature increases. The resistances will reach a minimum value that will be in lower irradiances for higher temperature levels. The minimum values can be found by using (42) and (43). The shadowed area in Fig. 4 is the 
Table 5: $M A E P_{a v}$ for $0 \leq V \leq V_{o c}$ and around the MPP. The average is computed for several irradiances.

\begin{tabular}{|c|c|c|c|c|c|c|c|c|c|c|c|c|}
\hline \multirow[t]{2}{*}{ Module } & \multicolumn{6}{|c|}{$0 \leq V \leq V_{o c}$} & \multicolumn{6}{|c|}{$V_{m p}-\Delta V<V<V_{m p}+\Delta V$} \\
\hline & A & B & $\mathrm{C}^{-}$ & $\mathrm{D}$ & Prop.1 & Prop. 2 & A & B & $\mathrm{C}$ & $\mathrm{D}$ & Prop.1 & Prop.2 \\
\hline KK280P & 0.9018 & 2.0244 & 1.2194 & 2.9094 & 0.7090 & 0.7086 & 0.6062 & 1.2475 & 1.0887 & 2.2887 & 0.6870 & 0.6697 \\
\hline JAP60S01 & 0.5161 & 1.2969 & 1.3790 & 3.0688 & 0.3767 & 0.3537 & 0.5377 & 1.5028 & 1.5417 & 2.9210 & 0.3301 & 0.2937 \\
\hline REC245PE & 1.3757 & 18.0345 & 14.9079 & 16.1447 & 1.4438 & 1.4019 & 0.6619 & 2.1668 & 2.1661 & 2.0229 & 0.5833 & 0.5948 \\
\hline PC1D & 0.0730 & 0.1962 & 0.0943 & 0.1238 & 0.0741 & 0.0751 & 0.0773 & 0.2710 & 0.0941 & 0.1649 & 0.0859 & 0.0892 \\
\hline M-60 & 0.3509 & 3.0948 & 1.2941 & 4.0221 & 0.2741 & 0.2497 & 0.5091 & 1.8660 & 1.2206 & 3.0480 & 0.1913 & 0.1626 \\
\hline
\end{tabular}

A: D. Soto solution [39]; B: Salam solution [34]; C: Villalva solution [41]; D: Sera solution [35].

Table 6: $M A E P_{a v}$ for $0 \leq V<V_{o c}$ and around the MPP. The average is computed for several Temperatures.

\begin{tabular}{|c|c|c|c|c|c|c|c|c|c|c|c|c|}
\hline \multirow[t]{2}{*}{ Module } & \multicolumn{6}{|c|}{$0 \leq V \leq V_{o c}$} & \multicolumn{6}{|c|}{$V_{m p}-\Delta V<V<V_{m p}+\Delta V$} \\
\hline & A & B & $\mathrm{C}$ & $\mathrm{D}$ & Prop.1 & Prop.2 & A & B & $\mathrm{C}$ & $\mathrm{D}$ & Prop.1 & Prop. 2 \\
\hline KK280P & 7.7474 & 3.8610 & 3.7828 & 7.6119 & 1.5745 & 1.5745 & 3.4641 & 3.4009 & 3.2835 & 6.8969 & 0.7464 & 0.7464 \\
\hline JAP60S01 & 2.2357 & 1.8638 & 2.5242 & 4.2067 & 1.4417 & 1.4417 & 1.0997 & 0.9957 & 1.1559 & 3.4388 & 0.5130 & 0.5130 \\
\hline REC245PE & 4.4608 & 16.5264 & 18.1640 & 18.2104 & 2.9509 & 2.9967 & 1.4043 & 3.7778 & 3.6210 & 3.8585 & 1.1483 & 1.1652 \\
\hline M-60 & 31.6314 & 8.4136 & 8.3520 & 10.9760 & 1.7181 & 1.7187 & 20.4090 & 3.2953 & 2.9785 & 6.3183 & 1.3026 & 1.3038 \\
\hline
\end{tabular}

A: D. Soto solution [39]; B: Salam solution [34]; C: Villalva solution [41]; D: Sera solution [35].

zone where $R_{s} \geq R_{s, \Delta n}$.

According to Fig. 3 and Tables 5-8, the inclusion of the approximations proposed in this paper, has resulted in improving the modelling accuracy throughout the whole range of the module voltage in a wide range of $\mathrm{T}$ and $\mathrm{G}$.

\section{Conclusion}

In this paper, new approximations for the series and shunt resistances of PV-modules were proposed. Besides, an expression for the bandgap energy considering the narrowing effect for heavy doping was used along with physical expressions for the remaining parameters. The proposed approximations were applied to the SD model. Furthermore, it was analysed with and without considering of the excess of carriers due to the PGE. The performance of the proposed models were compared with four well-known models using the I-V characteristics of different PC and MC PV modules. The results show the superiority of the proposed models in terms of accuracy in almost all the conditions of temperature and irradiance for the five modules analyzed. Although the proposed models contain a few tuning parameters, they vary within very narrow ranges, which allow to tune them even manually. However, to reach a good approximation for the tuning parameters, additional I-V curves are required, which can be obtained either from the $\mathrm{PV}$ datasheets or field measurements. In case the lower number of tuning parameters is desired, other information like doping concentration, doping distribution profile, and so on are required which are not normally provided by PV manufacturers. Thus, a satisfactory trade-off between accuracy and complexity is needed considering the model applications. Finally, considering the proposed physics-based modelling approach for representing the series and shunt resistances, the model has the potential to be used in extreme operating conditions through accurately considering the effect of temperature and irradiance on the PV resistances behavior. Besides, it can be used for degradation tracking, performance monitoring, PV design improvement, and other applications where the physical meaning of the $\mathrm{PV}$ parameters is of great importance.

\section{Acknowledgment}

This work was supported by the Mexican National Council of Science and Technology (CONACYT) [scholarship number 709940]; It was funded also by a Villum Investigator grant (no. 25920) from The Villum Fonden; And also sponsored by the Universidad de la Salle Bajío, Guanajuato, México.

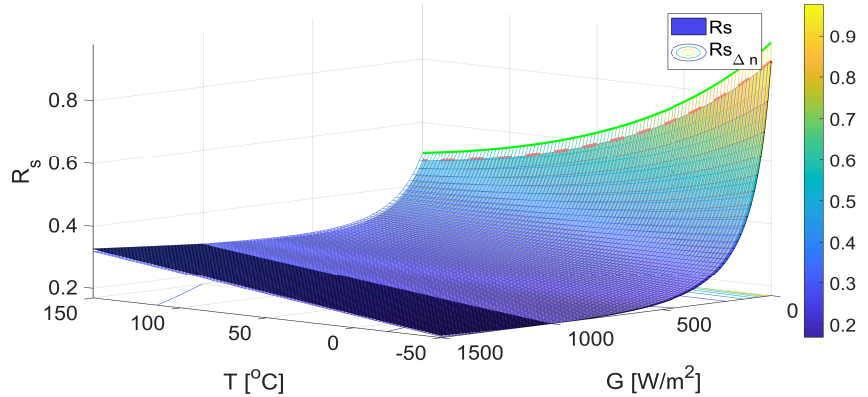

Figure 4: PV-module JAP60S01. $R_{s}$ : series resistance for model Prop. 1. $R_{s, \Delta n}$ : series resistance for model Prop. 2. Solid green-line: $R_{s, \Delta n}$ at $G=0$. Dashed red-line: $R_{s}$ at $G=0$. 
Table 7: Average RMSE of power for $0 \leq V \leq V_{o c}$ and around the MPP. The average is computed for several irradiances.

\begin{tabular}{|c|c|c|c|c|c|c|c|c|c|c|c|c|}
\hline \multirow{2}{*}{ Module } & \multicolumn{6}{|c|}{$\frac{1}{N} \sum_{\forall N} R M S E_{N} 0 \leq V \leq V_{o c}$} & \multicolumn{6}{|c|}{$\frac{1}{N} \sum_{\forall N} R M S E_{N} V_{m p}-\Delta V<V<V_{m p}+\Delta V$} \\
\hline & A & $\mathrm{B}^{N 2}$ & ${ }^{N} \mathrm{C}$ & $\overline{\mathrm{D}}$ & Prop.1 & Prop.2 & $\mathrm{A}^{\mathrm{N}}$ & $\nabla N \mathrm{~B}$ & $\mathrm{C}$ & $\mathrm{D}$ & Prop.1 & Prop. 2 \\
\hline KK280P & 1.3137 & 2.8525 & 1.5932 & 3.9898 & 1.0095 & 1.0111 & 0.7261 & 1.4178 & 1.2936 & 2.6797 & 0.7829 & 0.7621 \\
\hline JAP60S01 & 0.7141 & 1.6432 & 1.7735 & 4.6595 & 0.5725 & 0.5338 & 0.5597 & 1.5985 & 1.6444 & 3.7217 & 0.3637 & 0.3293 \\
\hline REC245PE & 1.6662 & 20.9017 & 17.4257 & 18.8156 & 1.6931 & 1.6378 & 0.6686 & 2.2575 & 2.2036 & 2.0675 & 0.5877 & 0.5988 \\
\hline M-60 & 0.4484 & 4.9867 & 1.6724 & 6.0045 & 0.4360 & 0.3891 & 0.5389 & 2.3731 & 1.3554 & 3.4790 & 0.2402 & 0.1983 \\
\hline
\end{tabular}

Table 8: Average RMSE of power for $0 \leq V \leq V_{o c}$ and around the MPP. The average is computed for several Temperatures.

\begin{tabular}{|c|c|c|c|c|c|c|c|c|c|c|c|c|}
\hline \multirow{2}{*}{ Module } & \multicolumn{6}{|c|}{$\frac{1}{N} \sum_{\forall N} R M S E_{N} 0 \leq V \leq V_{o c}$} & \multicolumn{6}{|c|}{$\frac{1}{N} \sum_{\forall N} R M S E_{N} V_{m p}-\Delta V<V<V_{m p}+\Delta V$} \\
\hline & A & $\mathrm{B}^{N-2}$ & $\mathrm{C}$ & $\overline{\mathrm{D}}$ & Prop.1 & Prop. 2 & A & B & C & D & Prop.1 & Prop.2 \\
\hline KK280P & 12.3243 & 4.9558 & 4.7456 & 9.8631 & 2.0624 & 2.0624 & 4.0382 & 3.5711 & 3.5024 & 7.4920 & 0.9169 & 0.9169 \\
\hline JAP60S01 & 3.2788 & 2.5414 & 3.5234 & 6.0553 & 2.7530 & 2.7530 & 1.3150 & 1.1779 & 1.4171 & 4.2604 & 0.5914 & 0.5914 \\
\hline REC245PE & 5.3401 & 19.2347 & 21.1252 & 20.9988 & 3.4061 & 72 & 1.4154 & 3.8430 & 3.6723 & 3.9007 & 1.1597 & 1.1768 \\
\hline PC1D & 2.5819 & 1.7673 & 1.8260 & 2.7153 & 0.5 & 0. & 0.6689 & 0.4025 & 0.4243 & 2.7067 & 0.1916 & 0.1916 \\
\hline M-60 & 47.7531 & 13.2887 & 12.8402 & 16.0188 & 2.5192 & 2.5198 & 23.7117 & 3.9325 & 3.3512 & 7.4103 & 1.4958 & 1.4969 \\
\hline
\end{tabular}

A: D. Soto solution [39]; B: Salam solution [34]; C: Villalva solution [41]; D: Sera solution [35].

\section{References}

[1] M. Akhsassi, A. E. Fathi], N. Erraissi, N. Aarich, A. Bennouna, M. Raoufi, and A. Outzourhit. Experimental investigation and modeling of the thermal behavior of a solar PV module. Solar Energy Materials and Solar Cells, 180:271 - 279, 2018.

[2] S. P. Aly, S. Ahzi, and N. Barth. An adaptive modelling technique for parameters extraction of photovoltaic devices under varying sunlight and temperature conditions. Applied Energy, 236:728 - 742, 2019.

[3] W. Ananda. External quantum efficiency measurement of solar cell. In 2017 15th International Conference on Quality in Research (QiR) : International Symposium on Electrical and Computer Engineering, pages 450-456, 2017.

[4] J. Arora, A. Verma, and M. Bhatnagar. Variation of series resistance with temperature and illumination level in diffused junction poly-and single-crystalline silicon solar cells. Journal of materials science letters, 5(12):1210$1212,1986$.

[5] Z. Ben Mahmoud, M. Hamouda, and A. Khedher. Effects of series and shunt resistances on the performance of PV panel under temperature variations. In 2016 International Conference on Electrical Sciences and Technologies in Maghreb (CISTEM), pages 1-7, 2016.

[6] A. N. Celik. Artificial neural network modelling and experimental verification of the operating current of monocrystalline photovoltaic modules. Solar Energy, 85(10):2507 - 2517, 2011.

[7] S. Chander, A. Purohit, A. Nehra, S. Nehra, and M. Dhaka. A study on spectral response and external quantum efficiency of mono-crystalline silicon solar cell. International Journal of Renewable Energy Research (IJRER), $5(1): 41-44,2015$.

[8] M. Chegaar and P. Mialhe. Effect of atmospheric parameters on the silicon solar cells performance. Journal of Electron Devices, 6(173-176), 2008.

[9] A. Coelho and R. Castro. Experimental validation of PV power output prediction models. In 2012 IEEE International Conference on Industrial Technology, pages 705-710, 2012.

[10] U. Department of Energy. Reference Air Mass 1.5 Spectra, 2020.

[11] B. K. Dey, I. Khan, N. Mandal, and A. Bhattacharjee. Mathematical modelling and characteristic analysis of Solar PV Cell. In 2016 IEEE 7th Annual Information Technology, Electronics and Mobile Communication Conference (IEMCON), pages 1-5, 2016.

[12] A. A. Elbaset, H. Ali, and M. A.-E. Sattar]. Novel seven-parameter model for photovoltaic modules. Solar Energy Materials and Solar Cells, 130:442 - 455, 2014.

[13] M. E. Elnagi Mahmoud, A. A. Zaki Diab, and D. A. Kotin. Simulation and Experimental Validation of TwoDiode Model of Photovoltaic (PV) Modules. In 2018 XIV International Scientific-Technical Conference on Actual Problems of Electronics Instrument Engineering (APEIE), pages 244-251, 2018.

[14] D. Fébba, R. Rubinger, A. Oliveira, and E. Bortoni. Impacts of temperature and irradiance on polycrystalline silicon solar cells parameters. Solar Energy, 174:628 - 639, 2018. 
[15] B. J. Hallam, P. G. Hamer, R. S. Bonilla, S. R. Wenham, and P. R. Wilshaw. Method of Extracting Solar Cell Parameters From Derivatives of Dark I-V Curves. IEEE Journal of Photovoltaics, 7(5):1304-1312, 2017.

[16] P. Hersch and K. Zweibel. Basic photovoltaic principles and methods. Technical report, Solar Energy Research Inst., Golden, CO (USA), 1982.

[17] H. Ibrahim and N. Anani. Variations of PV module parameters with irradiance and temperature. Energy Procedia, 134:276 - 285, 2017. Sustainability in Energy and Buildings 2017: Proceedings of the Ninth KES International Conference, Chania, Greece, 5-7 July 2017.

[18] K. Ishaque, Z. Salam, H. Taheri, and Syafaruddin. Modeling and simulation of photovoltaic (PV) system during partial shading based on a two-diode model. Simulation Modelling Practice and Theory, 19(7):1613 - $1626,2011$.

[19] S. Jain and D. Roulston. A simple expression for band gap narrowing (BGN) in heavily doped Si, Ge, GaAs and GexSi1- x strained layers. Solid-State Electronics, 34(5):453-465, 1991.

[20] M. Karamirad, M. Omid, R. Alimardani, H. Mousazadeh, and S. N. Heidari. ANN based simulation and experimental verification of analytical four- and five-parameters models of PV modules. Simulation Modelling Practice and Theory, 34:86 - 98, 2013.

[21] S. O. Kasap. Principles of electronic materials and devices. Tata McGraw-Hill, 2006.

[22] J. A. Kratochvil, W. E. Boyson, and D. L. King. Photovoltaic array performance model. United States. Department of Energy, 82004.

[23] H. P. D. Lanyon and R. A. Tuft. Bandgap narrowing in moderately to heavily doped silicon. IEEE Transactions on Electron Devices, 26(7):1014-1018, 1979.

[24] G. Li, C. Wang, J. Lu, and H. Zhang. Temperature impact on parameters of In0.3Ga0.7As PV cell under laser irradiation condition. AIP Advances, 9(9):095053, 2019.

[25] S. Li, W. Gong, X. Yan, C. Hu, D. Bai, and L. Wang. Parameter estimation of photovoltaic models with memetic adaptive differential evolution. Solar Energy, 190:465 - 474, 2019.

[26] Y. Mahmoud and E. El-Saadany. Accuracy improvement of the ideal pv model. IEEE Transactions on Sustainable Energy, 6(3):909-911, 2015.

[27] Y. Mahmoud, W. Xiao, and H. H. Zeineldin. A Simple Approach to Modeling and Simulation of Photovoltaic Modules. IEEE Transactions on Sustainable Energy, 3(1):185-186, Jan 2012.

[28] A. Mellit, M. Benghanem, and S. Kalogirou. Modeling and simulation of a stand-alone photovoltaic system using an adaptive artificial neural network: Proposition for a new sizing procedure. Renewable Energy, 32(2):285 - 313, 2007.

[29] E. Moshksar and T. Ghanbari. Adaptive Estimation Approach for Parameter Identification of Photovoltaic Modules. IEEE Journal of Photovoltaics, 7(2):614-623, 2017.

[30] A. J. Nascimento, M. C. Cavalcanti, F. Bradaschia, E. A. Silva, L. Michels, and L. P. Pietta. Parameter estimation technique for double-diode model of photovoltaic modules. In 2017 Brazilian Power Electronics Conference (COBEP), pages $1-6,2017$.

[31] P. K. Pandey and K. S. Sandhu. Multi diode modelling of PV cell. In 2014 IEEE 6th India International Conference on Power Electronics (IICPE), pages 1-4, 2014.

[32] C. S. Ruschel, F. P. Gasparin, E. R. Costa, and A. Krenzinger. Assessment of PV modules shunt resistance dependence on solar irradiance. Solar Energy, 133:35 - 43, 2016.

[33] K. Safa, K. Cyril, and R. H. E. Electrical Conduction in Metals and Semiconductors, pages 1-1. Springer International Publishing, Cham, 2017.

[34] Z. Salam, K. Ishaque, and H. Taheri. An improved two-diode photovoltaic (PV) model for PV system. In 2010 Joint International Conference on Power Electronics, Drives and Energy Systems 2010 Power India, pages 1-5, 2010 .

[35] D. Sera, R. Teodorescu, and P. Rodriguez. PV panel model based on datasheet values. In 2007 IEEE International Symposium on Industrial Electronics, pages 2392-2396, June 2007.

[36] S. Shongwe and M. Hanif. Comparative Analysis of Different Single-Diode PV Modeling Methods. IEEE Journal of Photovoltaics, 5(3):938-946, 2015. 
[37] E. A. Silva, F. Bradaschia, M. C. Cavalcanti, and A. J. Nascimento. Parameter Estimation Method to Improve the Accuracy of Photovoltaic Electrical Model. IEEE Journal of Photovoltaics, 6(1):278-285, 2016.

[38] E. A. Silva, F. Bradaschia, M. C. Cavalcanti, A. J. Nascimento, L. Michels, and L. P. Pietta. An Eight-Parameter Adaptive Model for the Single Diode Equivalent Circuit Based on the Photovoltaic Module's Physics. IEEE Journal of Photovoltaics, 7(4):1115-1123, 2017.

[39] W. D. Soto, S. Klein, and W. Beckman. Improvement and validation of a model for photovoltaic array performance. Solar Energy, 80(1):78 - 88, 2006.

[40] S. Sze and K. Ng. Physics of Semiconductor Devices. Wiley, 2006.

[41] M. G. Villalva, J. R. Gazoli, and E. R. Filho. Comprehensive Approach to Modeling and Simulation of Photovoltaic Arrays. IEEE Transactions on Power Electronics, 24(5):1198-1208, 2009.

[42] J. Wagner. Band-gap narrowing in heavily doped silicon at 20 and $300 \mathrm{~K}$ studied by photoluminescence. Phys. Rev. B, 32:1323-1325, Jul 1985.

[43] B. Yang, J. Wang, X. Zhang, T. Yu, W. Yao, H. Shu, F. Zeng, and L. Sun. Comprehensive overview of meta-heuristic algorithm applications on PV cell parameter identification. Energy Conversion and Management, 208:112595, 2020.

[44] K. Yu, X. Chen, X. Wang, and Z. Wang. Parameters identification of photovoltaic models using self-adaptive teaching-learning-based optimization. Energy Conversion and Management, 145:233 - 246, 2017.

[45] M. Zouine, M. Akhsassi, N. Erraissi, N. Aarich, A. Bennouna, M. Raoufi, and A. Outzourhit. Mathematical Models Calculating PV Module Temperature Using Weather Data: Experimental Study. In International Conference on Electronic Engineering and Renewable Energy, pages 630-639. Springer, 2018. 